\title{
ON $p$-GROUPS WHOSE MAXIMAL SUBGROUPS ARE ISOMORPHIC
}

\author{
AVINOAM MANN
}

(Received 14 December 1992)

\begin{abstract}
We give an improved bound for the order of a $p$-group enjoying the title property. We also point out relations between the upper central series of a $p$-group and of its maximal subgroups.

1991 Mathematics subject classification (Amer. Math. Soc.): $20 \mathrm{D} 15$.

Keywords and phrases: maximal subgroups of $p$-groups.

The problem of the structure of $p$-groups, all of whose maximal subgroups are isomorphic, was raised by Berkovich (see Problem 9 at the end of Berkovich [1]). In a recent paper Hermann [2] considers such groups. He shows that given a number $r$, there are only finitely many such groups, whose coclass is $r$ (we recall that the coclass $c c(G)$ of the $p$-group $G$ is $r=n-c$, where $|G|=p^{n}$, and $c=c l(G)$ is the class of $G$ ). Moreover, Hermann gives an explicit upper bound, roughly $(2 r)^{r}$, for $n$, given $r$. In this note we give a better bound, about $2^{r}$, and make some other remarks on the embedding of maximal subgroups of $p$-groups. As in Hermann [2] our main tool is comparing the upper central series of the $p$-group $G$ and of its maximal subgroups.
\end{abstract}

THEOREM 1. Let $G$ be a p-group of coclass $r$, all of whose maximal subgroups are isomorphic. Write $|G|=p^{n}, \operatorname{cl}(G)=c$. Then

$$
c \leq 2^{r}+1, \quad n \leq 2^{r}+r+1 .
$$

Hermann also shows, that for $r=1,2$ the best possible bounds for $n$ are 3 and 6, respectively, and that for $r=3$ one has $n \leq 13$. Our result is worse for $r=1,2$, but better for $r=3$. Still, it seems possible that a much better bound obtains. Indeed the theorem holds under weaker assumptions. It is obvious from the proof that it suffices to assume that $\left|Z_{i}(M)\right|$, for a maximal subgroup $M$ of $G$, depends only on $i$, not on $M$, and we can do with the following still weaker hypothesis:

(C) 1995 Australian Mathematical Society $0263-6115 / 95 \$ A 2.00+0.00$ 
$\left(^{*}\right)$ If $s \leq t<c l(G)$, and if $M_{1}, \ldots, M_{k}$ are all the maximal subgroups of $G$ such that $Z_{s}\left(M_{j}\right) \geq Z_{t}(G)$, then either the subgroups $Z_{s}\left(M_{j}\right), j=1, \ldots, k$ are all equal, or there exist two of them of which neither contains the other.

We start with a property that holds for all $p$-groups.

LEMMA 2. Let $G$ be any p-group, and let $M$ and $L$ be normal subgroups of $G$ satisfying $G=L M$ (in particular, $L$ and $M$ can be two distinct maximal subgroups). Then $Z_{i}(M) \cap Z_{j}(L) \leq Z_{i+j-1}(G)$.

PROOF. It is clear that $\left[x, x_{1}, \ldots, x_{i+j-1}\right]=1$, whenever $x$ belongs to the indicated intersection, and the elements $\left\{x_{u}\right\}$ belong to either $L$ or $M$.

REMARK. Dually, we have for the terms of the lower central series that $G_{i+j+1} \leq$ $M_{i+1} L_{j+1}$. This is just a restatement of the fact that if $M$ and $L$ are of classes $i$ and $j$, then $G$ is of class $i+j$ (at most).

LEMMA 3. Let $G$ satisfy $\left(^{*}\right)$. Then $Z(G)$ and $Z_{2}(G) / Z(G)$ cannot be both cyclic.

ProOF. Suppose that both $Z=Z(G)$ and $Z_{2}(G) / Z(G)$ are cyclic, and let $P / Z$ be the subgroup of order $p$ in $Z_{2}(G) / Z$. Then $M=C_{G}(P)$ is a maximal subgroup. Since $G / Z$ is not cyclic, there exist other maximal subgroups containing $Z$. Let $L$ be one of them. If $Z(L) \neq Z$, then $Z(L) \geq P$, so that $L \leq M$ and $L=M$. Thus $Z(L)=Z$ for all such maximal subgroups, so $\left(^{*}\right)$ is violated for $s=t=1$.

LEMMA 4. If $G$ satisfies $\left(^{*}\right)$ and is of maximal class, then $|G| \leq p^{3}$.

This follows from the previous lemma. For groups with all maximal subgroups isomorphic, Lemma 4 occurs already in Hermann [2], as does the special case $\left|Z_{2}(G)\right|=p^{2}$ of Lemma 3 .

PROOF OF THEOREM 1. Assume that $G$ satisfies ( $\left.{ }^{*}\right)$. Then Lemma 3 shows that we have $c c\left(G / Z_{2}(G)\right) \leq r-1$. Let $m$ be the largest integer for which $c c\left(G / Z_{t}(G)\right) \leq$ $r-m$, where $t=2^{m}$. If $m=r$ then $c c\left(G / Z_{t}(G)\right)=0$ implies $\left|G: Z_{t}(G)\right| \leq p$, which is possible only if $G=Z_{t}(G)$, so $\mathrm{cl}(G) \leq 2^{m}=2^{r}$. We thus may assume that $m<r$.

Let $M_{1}, \ldots, M_{k}$ be the maximal subgroups containing $Z_{t}(G)$, Suppose that the subgroups $Z_{t}\left(M_{i}\right)$ are not all identical. Then by $\left(^{*}\right)$ there exist indices $i, j$, such that neither $Z_{t}\left(M_{i}\right)$ nor $Z_{t}\left(M_{j}\right)$ contains the other. Write $N=Z_{t}\left(M_{i}\right) \cap Z_{t}\left(M_{j}\right)$, so that $Z_{t}(G) \leq N \leq Z_{2 t-1}(G)$, by Lemma 2 . Choose normal subgroups $K, L$, such that $N \leq K \leq Z_{t}\left(M_{i}\right), N \leq L \leq Z_{t}\left(M_{j}\right)$, and $|K: N|=|L: N|=p$. Then 
$|K L / N|=p^{2}$ and $K L / N \leq Z(G / N)$, so that $K L \leq Z_{2 t}(G)$ and $c c\left(G / Z_{2 t}(G)\right) \leq$ $c c(G / N)-1 \leq c c\left(G / Z_{t}(G)\right)-1 \leq r-(m+1)$, which contradicts the maximality of $m$, as $2 t=2^{m+1}$.

Therefore the subgroups $Z_{t}\left(M_{i}\right)$ are all the same, equal to $N$, say. Again $N \leq$ $Z_{2 t-1}(G)$. By the maximality of $m$ we have that $c c\left(G / Z_{2 t}(G)\right)=c c\left(G / Z_{t}(G)\right)$, which means that $\left|Z_{i+1}(G): Z_{i}(G)\right|=p$ for $t \leq i<2 t$. Then any normal subgroup between $Z_{t}(G)$ and $Z_{2 t}(G)$ is one of the subgroups $Z_{i}(G)$, and so we have $N=Z_{s}(G)$ for some $s$. Since for any maximal subgroup $M$ containing $N$ we have $Z_{i}(M / N)=Z_{t+i}(M) / N$, we see that $G / N$ also satisfies $\left(^{*}\right)$.

If $m=r-1$, then $G / N$ is of maximal class, hence of class 2 at most by Lemma 4 . Thus $\operatorname{cl}(G) \leq 2 t-1+2=2^{r}+1$. Finally, for $m \leq r-2$ we apply induction to conclude that $\operatorname{cl}(G) \leq 2 t-1+2^{r-m}+1=2^{m+1}+2^{r-m} \leq 2^{r}$. This ends the proof.

We now give two further propositions. The first, Proposition 6, is similar to Lemma 1 of Hermann [2], but we offer a different proof. Theorem 7 applies to all p-groups.

LEMMA 5. If $Z_{i}(G) \not M$ for some $i$ and some maximal subgroup $M$, then $Z_{i}(M)=$ $M \cap Z_{i}(G)$.

PROOF. We have $G=M Z_{i}(G)$. It is clear that $\left[x, x_{1}, \ldots, x_{i}\right]=1$, if $x \in Z_{i}(M)$, and $x_{u} \in M \cup Z_{i}(G)$. This proves that $Z_{i}(M) \leq M \cap Z_{i}(G)$, and the other inclusion is obvious.

PROPOSITION 6. Let $G$ be a p-group in which the ith terms of the upper central series of all maximal subgroups have the same order, for some $i$. Then $Z_{i}(G) \leq \Phi(G)$. In particular, if $\mathrm{cl}(G)=c$ and all maximal subgroups of $G$ are isomorphic, then $Z_{c-1}(G) \leq \Phi(G)$.

PROOF. There exists a maximal subgroup $M$ containing $Z_{i}(G)$, and then $Z_{i}(G) \leq$ $Z_{i}(M)$, so Lemma 5 shows that all other maximal subgroups also contain $Z_{i}(G)$.

THEOREM 7. Let $G$ be a p-group of class $c$ and coclass $r$. Then

(a) All but one at most of the maximal subgroups $M$ of $G$ satisfy $Z_{i}(M) \leq$ $Z_{2 i+r-1}(G)$, and unless perhaps $c \leq 2 i+r-1$, even $Z_{i}(M) \leq Z_{2 i+r-2}(G)$.

(b) If $k$ is the least index such that all but one of the maximal subgroups of $G$ satisfy $Z_{i}(M) \leq Z_{2 i+k-1}($ for some index $i)$, then $c c\left(G / Z_{2 i+k-1}(G)\right) \leq r-k$.

PROOF. (a) Let $M$ and $L$ be two maximal subgroups. We pass two central series $\left\{L_{j}\right\}$ and $\left\{M_{j}\right\}$ of $G$ with factors of order $p$ between $Z_{i}(M) \cap Z_{i}(L)$ and $Z_{i}(L)$ and 
$Z_{i}(M)$. Then $L_{j+1} M_{j+1} / L_{j} M_{j}$ is a central factor of order $p^{2}$ of $G$ and contributes 1 to the coclass of $G$. Therefore the length of one of these series at least is not more than $r$, which, together with Lemma 2 , shows that at least one of the subgroups $Z_{i}(M), Z_{i}(L)$ is contained in $Z_{2 i+r-1}(G)$. Moreover, if $M$, say, does not satisfy this inclusion, then letting $L$ vary over all other maximal subgroups of $\mathrm{G}$ establishes the first part of the claim.

(b) Keeping the same notation, we may assume that $M$ and $L$ are such that neither $Z_{i}(M)$ nor $Z_{i}(L)$ is contained in $Z_{2 i+k-2}(G)$. Then the length of both series $\left\{L_{j}\right\}$ and $\left\{M_{j}\right\}$ is at least $k$, so the central series $\left\{L_{j} M_{j}\right\}$ contributes at least $k$ to the coclass, leaving at most $r-k$ for the part above $Z_{2 i+k-1}(G)$. If here $k=r$, we obtain $c c\left(G / Z_{2 i+r-1}\right)=0$, which means that $G=Z_{2 i+r-1}(G)$.

It is certainly necessary to allow the exception in this proposition (consider groups with an abelian maximal subgroup). It seems desirable to study further the relations between the terms of the upper central series of a $p$-group and of its maximal subgroups. We tentatively offer the following definition.

DEFINITION. Let $G$ be a $p$-group. A maximal subgroup $M$ of $G$ is exceptional at the ith level, if there exists an index $k$, such that $Z_{i}(L) \leq Z_{k}(G)$, for all maximal subgroups $L$ different from $M$, but $Z_{i}(M)$ does not satisfy the same inclusion. $M$ is exceptional, if it is exceptional at some level.

EXAMPLE 1. Suppose both $Z(G)$ and $Z_{2}(G) / Z(G)$ are cyclic, and let $P / Z(G)$ be the unique normal subgroup of order $p$ of $G / Z(G)$. Then $C_{G}(P)$ is a maximal subgroup which is exceptional at the first level.

EXAMPLE 2. Let $G$ be of maximal class. Then most maximal subgroups are also of maximal class, excepting always one maximal subgroup $G_{1}$, and sometimes another one, say $S$. The subgroup $G_{1}$, which is the centraliser of all factors $Z_{i+2}(G) / Z_{i}(G)$, for $i \geq 1$, has its class bounded by some function of $p$, while $S$, which is the centraliser of $Z_{2}(G)$ (if this centraliser is different from $G_{1}$ ), and which, given $p$, exists in only finitely many cases, has a centre of order $p^{2}$ and all other upper central factors of order $p$ (for these properties of groups of maximal class, see for example Huppert [3, Section III.14]. Thus for all maximal subgroups $M$, excepting $G_{1}$, we have $Z_{i}(M) \leq Z_{i+1}(G)$, and if we except also $S$, even $Z_{i}(M) \leq Z_{i}(G)$. It follows that $G_{1}$ is exceptional at all levels $i>1$, while $S$ (when it exists) or $G_{1}$ is exceptional at level 1 .

PROBLEM. Study $p$-groups having no exceptional maximal subgroups. For example are there only finitely many such groups of a given coclass?

Finally, we remark that it is quite possible for many, but not all, of the maximal subgroups of a $p$-group $G$ to be isomorphic, without getting any restriction of the type 
of Theorem 1. Suppose, for example, that $G$ is a 2-generator maximal subgroup of a p-group $H$, and that not all maximal subgroups of $G$ are normal in $H$. Then of the $p+1$ maximal subgroups of $G$, one is normal in $H$, and the other $p$ are conjugate to each other, hence isomorphic. If $H$ is of maximal class and order at least $p^{4}$, it has a unique normal subgroup of index $p^{2}$, and $p$ of its maximal subgroups have two generators, hence satisfy our assumptions. Moreover, at least $p-1$ of these maximal subgroups are themselves of maximal class, hence for all orders there exist groups of coclass 1 with all maximal subgroups but one isomorphic. This example, as well as Theorem 7, suggest that it may be of interest to consider properties that are shared by all but one of the maximal subgroups of a $p$-group.

\section{References}

[1] Ya. G. Berkovich, 'A generalization of theorems of Ph. Hall and Blackburn and an application to non-regular $p$-groups', Math. USSR (Izv.) 5 (1971), 815-844.

[2] P. Z. Hermann, 'On finite $p$-groups with isomorphic maximal subgroups', J. Austral. Math. Soc. (Series A) 48 (1990), 199-213.

[3] B. Huppert, Endliche Gruppen I (Springer, Berlin, 1967).

Institute of Mathematics

Hebrew University

Givat Ram, Jerusalem 91904

Israel 